\title{
Systematic conservation Planning
}

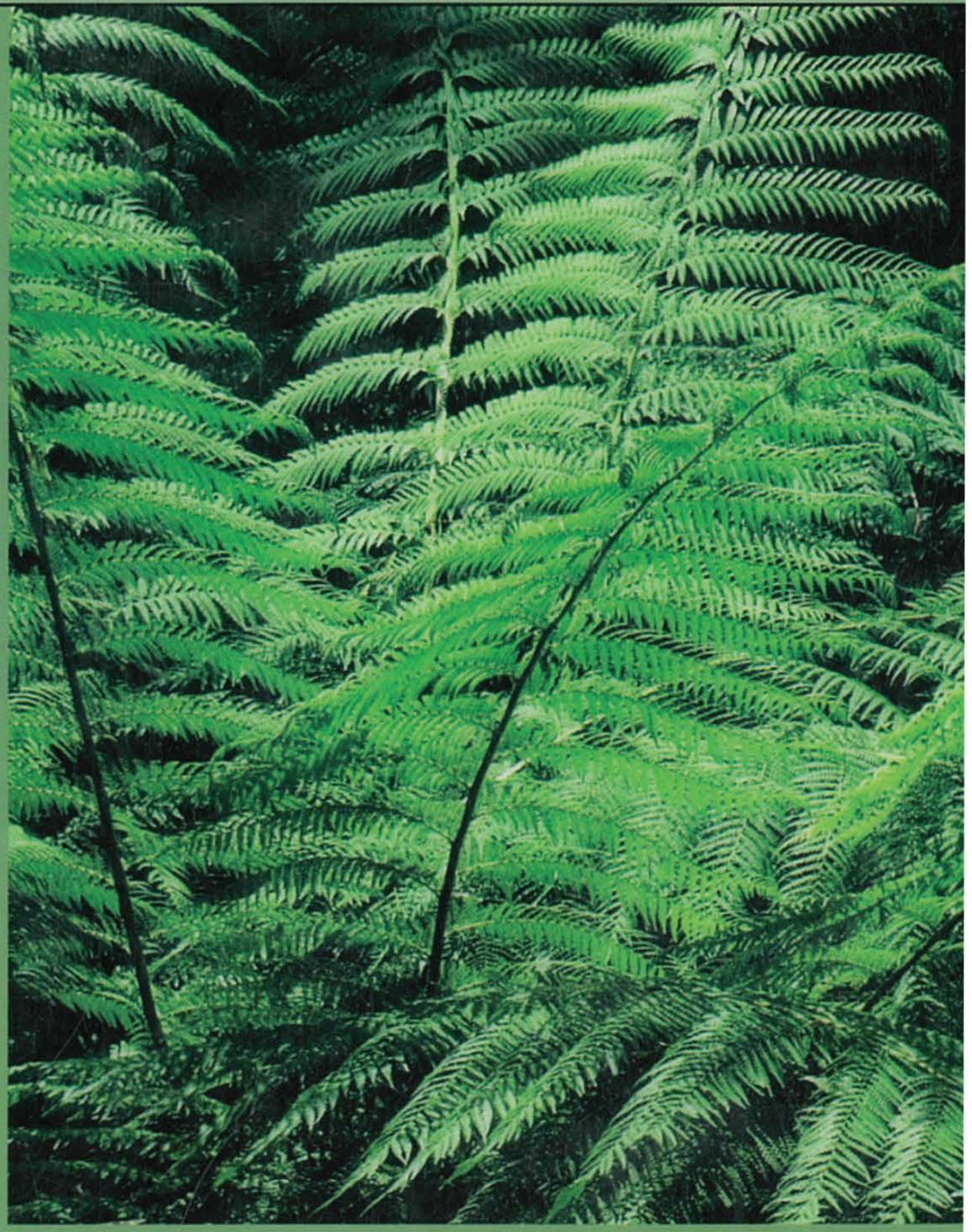




\section{Systematic Conservation Planning}

Systematic Conservation Planning provides a clear, comprehensive guide to the process of deriving conservation area networks that will represent biodiversity in cost-effective ways. The measurement of biodiversity, the design of field collections to sample biodiversity and data treatment methods together provide baseline information on the distribution patterns of biodiversity. The book then describes methods for identifying biodiversity priority areas underpinned by the concept of complementarity. It goes on to describe how biodiversity targets can be set and how multi-criteria analyses can incorporate costs and other constraints into the area selection process, enabling trade-offs between production and protection to be made. The book finishes with a series of case studies and a conclusion that emphasizes the socio-economic and cultural contexts in which conservation planning takes place.

A clear procedure is provided for identifying biodiversity priority areas, underpinned by current best science practice and sound common sense, making this volume of interest not only to graduate students and academic conservation biologists, but also planners and decision-makers dealing with natural resource management together with conservation NGOs.

Chris MARgules is Executive Director at the Melanesia Centre for Biodiversity Conservation, Conservation International.

SAHotra SARKar is a Professor in the Section of Integrative Biology at the University of Texas at Austin. 


\title{
ECOLOGY, BIODIVERSITY AND CONSERVATION
}

\author{
Series editors \\ Michael Usher University of Stirling, and formerly Scottish Natural Heritage \\ Denis Saunders Formerly CSIRO Division of Sustainable Ecosystems, Canberra \\ Robert Peet University of North Carolina, Chapel Hill \\ Andrew Dobson Princeton University \\ Editorial Board \\ Paul Adam University of New South Wales, Australia \\ H. J. B. Birks University of Bergen, Norway \\ Lena Gustafsson Suedish University of Agricultural Science \\ Jeff McNeely International Union for the Conservation of Nature \\ R. T. Paine University of Washington \\ David Richardson University of Cape Town \\ Jeremy Wilson Royal Society for the Protection of Birds
}

The world's biological diversity faces unprecedented threats. The urgent challenge facing the concerned biologist is to understand ecological processes well enough to maintain their functioning in the face of the pressures resulting from human population growth. Those concerned with the conservation of biodiversity and with restoration also need to be acquainted with the political, social, historical, economic and legal frameworks within which ecological and conservation practice must be developed. This series will present balanced, comprehensive, up-to-date and critical reviews of selected topics within the sciences of ecology and conservation biology, both botanical and zoological, and both "pure" and "applied". It is aimed at advanced final-year undergraduates, graduate students, researchers and university teachers, as well as ecologists and conservationists in industry, government and the voluntary sectors. The series encompasses a wide range of approaches and scales (spatial, temporal, and taxonomic), including quantitative, theoretical, population, community, ecosystem, landscape, historical, experimental, behavioral and evolutionary studies. The emphasis is on science related to the real world of plants and animals, rather than on purely theoretical abstractions and mathematical models. Books in this series will, wherever possible, consider issues from a broad perspective. Some books will challenge existing paradigms and present new ecological concepts, empirical or theoretical models, and testable hypotheses. Other books will explore new approaches and present syntheses on topics of ecological importance.

The Ecology of Plytoplankton

C. S. Reynolds

Invertebrate Conservation and Agricultural Ecosystems

T. R. New

Risks and Decisions for Conservation and Environmental Management

Mark Burgman

Nonequilibrium Ecology

Klaus Rohde

Ecology of Populations

Esa Ranta, Veijo Kaitala and Per Lundberg

Ecology and Control of Introduced Plants

Judith H. Myers, Dawn Bazely 
Systematic

Conservation

Planning

CHRISTOPHER R. MARGULES

Conservation International and CSIRO Sustainable Ecosystems,

Tropical Forest Research Centre, Atherton, Queensland

SAHOTRA SARKAR

Section of Integrative Biology, and Department

of Philosophy, University of Texas at Austin 
CAMBRIDGE UNIVERSITY PRESS

Cambridge, New York, Melbourne, Madrid, Cape Town, Singapore, São Paulo

Cambridge University Press

The Edinburgh Building, Cambridge CB2 8RU, UK

Published in the United States of America by Cambridge University Press, New York

www.cambridge.org

Information on this title: www.cambridge.org/9780521878753

(C) C. Margules and S. Sarkar 2007

This publication is in copyright. Subject to statutory exception and to the provisions of relevant collective licensing agreements, no reproduction of any part may take place without the written permission of Cambridge University Press.

First published 2007

Printed in the United Kingdom at the University Press, Cambridge

A catalog record for this publication is available from the British Library

ISBN 978-0-521-87875-3 hardback

ISBN 978-0-521-70344-4 paperback

Cambridge University Press has no responsibility for the persistence or accuracy of URLs for external or third-party internet websites referred to in this publication, and does not guarantee that any content on such websites is, or will remain, accurate or appropriate. 


\section{Contents}

Acknowledgments

page viii

1 Introduction 1

1.1 Conservation-area networks 2

1.2 What do we mean by biodiversity? 5

1.3 Systematic conservation planning 8

1.4 Summary 16

2 Biodiversity surrogates 19

2.1 True and estimator surrogates 20

2.2 Establishing the adequacy of an estimator-surrogate set $\quad 22$

2.3 Traditional species-based surrogates 27

2.4 Systematic surrogate sets 30

2.5 Surrogacy and spatial scale $\quad 42$

2.6 A protocol for the identification of an adequate surrogate set 43

2.7 Diversity of ecological processes 44

2.8 Summary 45

3 Data collection $\quad 47$

3.1 Areas and features $\quad 47$

3.2 Sources of data 51

3.3 Collecting new data with field surveys 56

3.4 Summary 76

4 Data treatments $\quad 79$

4.1 Conceptual framework 79

4.2 Multi-variate pattern analysis 83

4.3 Heuristic models 90

4.4 Regression models 93 
4.5 Machine-learning methods 100

4.6 Summary 103

5 Conservation-area networks 105

5.1 The role of conservation-area networks 105

5.2 The goals of networks: representativeness, persistence and economy 108

5.3 Selecting networks: complementarity 112

5.4 Selecting networks: rarity and adjacency 120

5.5 Subsidiary goals: flexibility, transparency, modularity, genericity and irreplaceability 121

5.6 Algorithms for the selection of networks 124

5.7 The trouble with scoring and ranking procedures

5.8 Summary

6 Persistence and vulnerability 131

6.1 Incorporating biological processes 132

6.2 Viability analysis 135

6.3 Targets for representation 151

6.4 Formal decision analysis $\quad 162$

6.5 Summary 166

7 Satisfying multiple criteria 169

7.1 Iterative- and terminal-stage procedures 171

7.2 The valuation framework 172

7.3 Non-dominated alternatives 176

7.4 Refining non-dominated sets 180

$\begin{array}{llr}7.5 & \text { Sensitivity analysis } & 188\end{array}$

7.6 Summary 194

8 Systematic conservation plans 197

8.1 Complementarity by inspection in the Nullarbor region, Australia 197

8.2 Complementarity using species records in Québec 205

8.3 A marine conservation plan for the California Channel Islands, United States 209

8.4 A conservation plan for the Cape Floristic Region of South Africa 214

8.5 A conservation plan for Papua New Guinea 219

8.6 Summary 225 
Contents . vii

9 Conclusions 227

9.1 Coping with uncertainty 228

9.2 Practicing conservation science in a complex world 231

9.3 Future directions $\quad 233$

9.4 Summary 238

References $\quad 239$

Index 265

The colour plates are situated between pages 130 and 131 . 


\section{Acknowledgments}

The collaboration from which this book emerged began at the Wissenschaftskolleg zu Berlin where both authors were fellows in the mid1990s. We thank the Kolleg for its support. We also thank Paul Griffiths of the University of Queensland Biohumanities Project for providing us with hospitality while we finished the book. A huge thanks also to Elva Castino for all her help with finalizing material and getting everything ready for publication.

The text of the first two parts of Chapter 3 draws heavily on material from Margules et al. (2002) and Williams et al. (2002). The text of Chapter 7 draws heavily on Moffett \& Sarkar (2005) and Moffett et al. (2006). We thank Jim Dyer, David Hilbert, Alex Moffett, Bob Pressey and Paul Williams for permission to use this material, as well as for many comments and discussions over the years.

For commenting on the entire manuscript we thank Trevon Fuller, Justin Garson, David Grice, James Justus and Liz Poon. For commenting on individual chapters we thank Mark Burgman, Dan Faith, Simon Ferrier, Nick Nicholls and Michael Usher.

For critical comment and discussion on many of the ideas in this book, sometimes over many years, we would also like to thank Anshu Aggarwal, Mike Austin, Mark Boyce, Helen Cortes-Burns, Chris Humphries, Patricia Illoldi, Chris Johnson, Chris Kelley, Vanessa Lujan, Camille Parmesan, Samraat Pawar, Hugh Possingham, Karen Richardson, Victor Sánchez-Cordero, Helen Sarakinos, Mike Singer, Dick Vane-Wright, Paul Walker, Kristen Williams and Paul Williams.

CRM would like to record a very special thanks to Janice Marion Margules, 1955-94, for her tireless support and encouragement of the ideas embodied in this book. 


\section{$1 \cdot$ Introduction}

The earth is experiencing a wave of extinctions possibly unprecedented in recent evolutionary history. The ecological advantage conferred upon humans by the evolution of reason and language has enabled us to obtain competitive dominance over all other species and expand our realized niche to include components of almost all habitats on the planet. We have simplified complex and diverse natural systems and processes for the production of food and shelter, leading to massive extinctions of species (Taylor 2004). The previous six mass extinctions caused by cataclysmic events decimated life on the planet and the current wave shows signs of approaching this level. These previous waves of extinction occurred over millennia; brief in geological time but long in ecological time. Life forms had the opportunity to adapt and evolve. Many species became extinct, but many others prospered and new species evolved. The current wave is not yet 300 years old; a brief aberration even in ecological time. Though humans have undoubtedly changed ecological processes and caused extinctions for longer than that, it was not at the current scale. Perhaps what is most troubling about the current wave of extinctions is that, unlike previous events, it is taking place because of a type of irreversible transformation of the land and even the oceans that is likely to make them unsuitable as habitat for many forms of life into the distant future.

The task for conservation biology is to halt the current extinction wave and chart a course for a future which includes biological diversity not only for its direct contribution to human welfare as a resource, but also because it appeals to important human values (Norton 1987; Sarkar 2005). In that future, nature conservation must exist alongside nature exploitation, not instead of it. Better still, the protection of biodiversity should become integrated into natural resource management that has sustainable livelihoods as an equally important goal. The protection of biodiversity will not win out in direct competition with other needs and aspirations of people (Rosenzweig 2003). Indeed, the research frontiers 


\section{2 . Introduction}

in conservation biology clearly must link a biophysical understanding of the world around us with a socio-economic understanding of what drives decisions on natural resource use, as well as an understanding of natural resource governance mechanisms and the institutional arrangements that societies use to regulate the exploitation of natural resources. This task of planning for the future is made even more complex by global environmental changes, especially climate change, the detailed consequences of which we can only dimly foresee, and even that only for a very few species. We will return to this issue of linking socio-economic goals with biodiversity conservation in Chapters 5 through 9, especially Chapters 7 , 8 and 9. The focus in Chapters 2, 3 and 4 is on methods for deriving biodiversity data sets suitable for use in conservation planning.

\subsection{Conservation area networks}

Central to the conservation of biological diversity is the establishment of networks of conservation areas which are managed to minimize the risk of extinction. These are often called protected areas, but we use the term conservation areas to include all areas that perform a conservation function, whether they are strictly protected or not (Sarkar 2003). These are the priority areas for the allocation of scarce biodiversity management resources. Systematic conservation planning consists of the use of specific protocols to identify such priority areas and separate them from processes which threaten their persistence. Identifying and securing biodiversity priority areas will not in itself protect biodiversity, but networks of priority areas in each region of the world should form the framework upon which other conservation actions build.

The key concept underpinning systematic conservation planning is complementarity. Complementarity is a measure of the contribution an area in a planning region makes to the full complement of biodiversity features: species, assemblages, ecological processes, etc. As discussed below and in later chapters, we can never know the full complement of biodiversity features; consequently we must use partial measures or surrogates and we must set goals for the representation of these surrogates. Complementarity can then be measured by the contribution an area makes to the conservation goal. For example, pretend that the conservation goal is to represent at least one population of all of the vascular plant species that occur in a planning region in a minimal set of biodiversity priority areas. The first area we choose might be the one with the most species. The next area we choose will be the one with the most 
species that were not represented in the first area and the next area after that will be the one with the most remaining unrepresented species, and so on until all species are represented. There are two important points to note about this process. The first is that as areas are added to the set, the contribution that remaining areas make to the goal changes. This is because some of the species in those areas may have already been contributed by other areas previously selected for the set. The second is that areas with the highest complementarity will not necessarily be those with the most species. In this example, complementarity is measured at each step as the number of unrepresented species. If an area has few species, but they do not occur widely in the planning region, it may have higher complementarity than an area with many species that are widespread throughout the planning region (depending on which species occur in areas that have already been selected). Thus, species richness cannot be used to measure complementarity. There are many variations on the simple area selection process outlined above and many of these are described later in the book. As it turns out, for example, it is often more economical to begin the area-selection process by selecting that area with the rarest species on it and then the one with the highest number of rarest unrepresented species, and so on until all species are represented (Sarkar et al. 2002). Vane-Wright et al. (1991) coined the term "complementarity," but it appears to have been independently discovered at least four times (Justus \& Sarkar 2002), twice in Australia (Kirkpatrick, 1983; Margules \& Nicholls 1987; Margules et al. 1988), in the United Kingdom (Ackery \& Vane-Wright 1984) and in South Africa (Rebelo \& Siegfried 1990). Complementarity is described in detail in Chapter 5 (Section 5.4).

Systematic conservation planning is usually implemented with software tools using digital georeferenced data sets and area selection algorithms. Algorithmic approaches ensure that planning exercises are standardized and repeatable - this is the most important sense in which conservation planning is systematic. However, this numerical computerbased approach has led to some criticisms, in particular that large data sets are needed to run the software (Redford et al. 1997; Prendergast et al. 1999), that local expertise is not consulted and incorporated (Redford et al. 1997) and that they are expensive and money could better be spent acquiring reserves (Prendergast et al. 1999). Any reading of the conservation planning literature will show that these criticisms are unjustified. There is no substitute for local expertise, which should always be consulted. In fact, systematic conservation planning works best when local experts are the ones doing the planning. The methods are 
inexpensive, can be used without computers and with minimal data. It is just that computers speed up the process and enable large data sets to be used efficiently when they are available. Also, the recent growth in biological and environmental databases, in part due to rapid improvements in remote sensing and modeling, means that there is now almost no terrestrial region on Earth with so little data that systematic planning tools cannot improve policy formulation.

Where population density is high and land is in private ownership, conservation agencies have, in the past, found it necessary to take opportunities whenever and wherever they occurred believing that when land is privately owned there is no opportunity for a more strategic systematic approach. However, if conservation NGOs (non-government organizations) such as The Nature Conservancy in the United States and Bush Heritage in Australia are purchasing land, as they currently are, they need to know what the genuine biodiversity priorities are or they will not make best use of their funds. If they purchase the cheapest land or just whatever becomes available they will not make the contributions to biodiversity conservation that they could make, and which are sorely needed. As several past studies have shown, ad hoc conservation-area selection has been remarkably cost-ineffective (Pressey et al. 1996; Pressey \& Cowling 2001). This is because conservation areas all over the world contain a biased sample of biodiversity from ecosystems and habitats that were selected because they are remote and inaccessible, or they are unfit for alternative uses such as agriculture, which means they are cheap. The identification of biodiversity priority areas should be based on good science and sound common sense and not on such ad hoc grounds. Some priority areas will inevitably be expensive. But even if they prove too expensive to buy, we should know about them. They might become subject to policy change and/or management agreements in future. Planning tools support that process. They do not replace it. Conservation planning is a dynamic iterative process and these planning tools are designed for decision support to help local experts identify good policy options, not formulate policy on their behalf.

In the United States, Texas provides a striking example of the potential continued inefficient use of resources when conservation policy is not determined by systematic planning (Alford 2005). In 2003 tax exemptions given to landowners for maintaining or restoring their land as wildlife habitat were estimated to be over $\$ 1$ billion. Land management options included habitat control (such as clearing juniper overgrowth), erosion control, control of predators (such as feral cats or dogs), providing water 
resources, providing supplemental food for wild animals, growing nutritional plants, providing animal shelters (such as leaving dead trees for bird nests) and conducting animal censuses. There is no coordination among efforts of individual landowners. Although those who receive the tax break have to formulate explicit wildlife management plans, these do not have to be filed with any state agency, but only be appraised by county officials who need not have any wildlife expertise. There is no monitoring to determine whether plans are successful in promoting wildlife. In some cases anecdotal evidence suggests no change: the deer population growth rate remained the same before and after the policy was introduced in 1995. In other cases, there may be some success: while the decline of wild turkey populations was $54 \%$ in the five years preceding 1995 , it was only $9 \%$ in the subsequent five years. Given the lack of coordination and monitoring it is impossible to evaluate what is being conserved or restored and how effectively. Perhaps more importantly, there is no prioritization of land on the basis of its potential contribution to a biodiversity goal, given appropriate management, and therefore no prioritization of the landowners who should receive these tax breaks. This is the type of problem that systematic conservation planning aims to mitigate.

In some places where land is privately owned and there are few opportunities for biodiversity protection using traditional approaches, marketbased instruments are being tested and evaluated. In a recent pilot study in the highly fragmented landscape of the Western Australian wheatbelt called "Auction for Landscape Recovery" (Gole et al. 2005), landowners were invited to nominate portions of their holdings and propose management actions for these portions and then bid for funds to implement those actions. Bids were judged on two criteria. One was the contribution the nominated areas made to a regional biodiversity goal, i.e., complementarity, and the other was the likely success of the proposed management actions. This is an example of how the systematic approach is beginning to find wide application in the development and implementation of conservation policies and practices.

\subsection{What do we mean by biodiversity?}

Biodiversity consists of the biological variety bequeathed to us by evolutionary processes over millennia. It is what we have to conserve if we do not want to squander this inheritance. The biological realm is characterized by variability and complexity at every level of structural, taxonomic and functional organization. The term "biodiversity" was introduced in 
the mid 1980s as a contraction of "biological diversity" to refer to the totality of this variability (Takacs 1996). From a biological perspective, all such diversity is important because it provides the raw material for evolution. Ideally, all of it should be conserved.

Ecosystem processes such as nutrient cycling, the movement of water and energy and the dispersal of propagules are necessary for the persistence of biodiversity, but in this book we will restrict the definition of "biodiversity" to biological patterns such as the distribution patterns of biological entities and dynamic behavioral phenomena such as migrations. Two hierarchical schemes are used for the classification of biological entities (Sarkar 1998). One is a spatial (or ecological) hierarchy starting with molecules and macromolecules, then cell organelles, cells, individuals, populations and metapopulations, communities, ecosystems and ultimately the biosphere. The second is a taxonomic hierarchy from alleles to loci, linkage groups, genotypes, subspecies, species, genera, families, orders, classes, phyla and kingdoms. Both hierarchies reflect evolutionary history and are constrained by evolutionary mechanisms. Since the future for biodiversity is dependent on evolutionary processes, understanding the relationships between these two hierarchies and phylogeny is also necessary for the formulation of successful conservation strategies. In practice we never know these relationships fully but must proceed anyway, accepting that conservation priorities and strategies will change as knowledge accumulates.

There are two points to note about both hierarchies. The first is that they are not clean and tidy in the sense that biological entities fall neatly into well-defined classes without exception. The second is that classes at all levels in each hierarchy are heterogeneous: there is variety within each class at every level. The variety of viable biological configurations at all hierarchical levels is extremely large, currently unknown, and probably unmeasurable. This second point is almost trivial, but emphasizes the complexity of biodiversity. Almost any two populations, even of the same subspecies, differ in their genetic profiles. Except for some clonal organisms, almost any two individuals of the same species have different genotypes. There are virtually no two identical ecological communities, and so on. The first point is equally important when we consider how to describe biodiversity. While some entities such as organelles and cells are reasonably well defined, examples such as fungi, symbionts and clonal organisms show that even biological "individuals" are not always precisely defined. Asexual species are notoriously difficult to define and even sexual species, usually defined by the ability to interbreed and produce 
offspring (Mayr 1957), cause problems, for instance in the case of socalled "ring species." In such species, "rings" are composed of circular geographically successive populations that evolved from a single ancestral population. Each pair of neighboring populations can interbreed except for the two terminal ones which, by definition, should now be regarded as different species. Examples include the greenish warbler, Phylloscopus trochiloides, populations surrounding Tibet and the salamander, Ensatina eschscholtzii, surrounding the central valley of California (Wake 2001).

As we will discuss in detail in Chapter 2, biodiversity, described in such a complex way, is impossible to estimate or quantify in the field; it is not an operational concept (Sarkar 2002; Sarkar \& Margules 2002). In practice, the concept of biodiversity has often been simplified to refer to diversity at three levels of organization: genes (alleles), species and ecosystems (e.g., Meffe \& Carroll 1994; Vermeulen \& Koziell 2002 and see Lindenmayer \& Burgman 2005 for a detailed discussion of biodiversity at different organizational and functional levels). Any such definition is necessarily partly conventional. However, that does not mean that it must be arbitrary. Such a definition is conventional because we know that it does not include all of what we mean by diversity. For instance, the definition of biodiversity as diversity of alleles, species and ecosystems excludes interspecific hybrids. More importantly, it excludes biological phenomena such as the annual migrations of north American monarch butterflies (Danaus plexippus), which may be endangered and deserving protection (Brower \& Malcolm 1991; see Chapter 2). Nevertheless, the definition is not arbitrary because focusing conservation efforts on genes, species and ecosystems will protect much of the diversity within species, taxonomic diversity at levels higher than species and many communities (see Chapter 2).

As Austin and Margules (1986) first explicitly pointed out, the concept of biodiversity must be operationalized through the use of "surrogates," features of the landscape such as the presence of species or other taxa, habitat type, etc., that can in principle be quantified and assessed in the field. Surrogates that are used to represent biodiversity in its full generality we call "true" surrogates. For example, "biodiversity in general" is often thought to be adequately represented by species diversity. The identification and adoption of true surrogates operationalizes the concept of biodiversity for systematic conservation planning. But, as we noted earlier, any choice of a true surrogate set is partly conventional. Moreover, since general biodiversity cannot be quantified, the success of a true surrogate set in representing general biodiversity also cannot be quantitatively 
assessed. What we must do is give good reasons for what we choose to be the true surrogate set in a given planning context. Quite often, we are not in a position even to assess the full distribution of a true surrogate set. For instance, if we take all species within even well-documented taxa such as mammals and birds, there are many areas of the world for which we will not have all the distributional data we need (see Chapter 3). In many situations we may not even be able to model such distributions completely (see, however, Chapter 4). We then have to use "estimator surrogates" for biodiversity; those features which we can in practice quantify and assess in the field. The question whether an estimator surrogate set adequately represents a true surrogate set is amenable to quantitative assessment, as we discuss in detail in Chapter 2.

\subsection{Systematic conservation planning}

Systematic conservation planning is a structured step-wise approach to mapping conservation area networks, with feedback, revision and reiteration, where needed, at any stage. Though prioritizing new areas for conservation is central to systematic conservation planning, this process does not ignore or throw away, literally or metaphorically, existing conservation areas or networks. In almost all regions of the world, there is a heritage of conservation areas, which more than likely have been accumulated opportunistically and are therefore unrepresentative of regional biodiversity. Systematic planning normally accepts these imperfect networks and maps onto, or builds on, what already exists, with the objective of transforming them into better networks. In addition, analyses of the extent to which existing conservation areas contribute to regional biodiversity goals might provide options for future rationalization. For example, it may be possible to trade existing conservation areas making low contributions to biodiversity representation for new areas that would make higher contributions.

Margules and Pressey (2000), Groves et al. (2002), Cowling and Pressey (2003) and Sarkar (2004) identified several distinguishing characteristics of systematic conservation planning. In the first place, it requires the identification and engagement of stakeholders. The people who rely on or influence the use of natural resources in the planning region must be party to the planning process or it will fail. This point deserves emphasis: far too often conservation policies have failed because people who have a stake in the land or water that is the subject of those policies have not been consulted (Sarkar 1999; Justus \& Sarkar 2002). Sometimes, 
when the planners have come from international organizations or distant national governments, this has led to accusations of paternalism, colonialism and other ideological remnants of the Northern colonial era (Guha 1989). Recent reports have documented the creation of "conservation refugees," people involuntarily displaced by conservation policies such as the creation of reserves and the abrogation of traditional resource-use rights such as hunting (Dowie 2005). Throughout this book we emphasize the point that systematic conservation planning must be viewed as part of social policy which explicitly recognizes and addresses these issues.

Systematic conservation planning also requires that clear choices are made about how biodiversity is to be measured and mapped. This is partly the surrogacy issue raised in Section 1.2 above and discussed in detail in Chapter 2. But it also includes collecting data, building and managing data sets and databases, and carrying out data treatments to derive the chosen surrogates. These issues are discussed in Chapters 3 and 4. Similarly, clear goals have to be set and preferably then translated into explicit measurable conservation targets. Next, the extent to which conservation goals have been met in existing reserves or priority areas must be recognized so that the simple explicit methods described in Chapter 5 can be used to identify new priority areas to complement existing conservation areas in achieving the set goals. Finally, explicit criteria for implementing planning choices on the ground have to be formulated and applied, objectives for individual conservation areas have to be set, the achievement of those objectives has to be monitored and appropriate management actions have to be taken to ensure continued contribution of individual conservation areas to overall conservation goals. This book addresses all of these issues, though Chapter 6 is the only one to focus on the last, while also addressing issues of threat and vulnerability at all stages in the process.

Table 1.1, modified from Margules and Pressey (2000) and Sarkar (2004), describes this overall approach in eleven more-detailed stages. The first stage is stakeholder engagement. Stakeholders will often be local residents, farmers or pastoralists, but can also include government agencies responsible for managing natural resources such as water and forests, nongovernment organizations (NGOs), including conservation NGOs, both local and global, and industries, e.g., mining and agri-businesses. Stakeholders include all those people who have decision-making powers over a region, all those who will be affected by the conservation plans that are formulated, those with scientific or other expertise about the region and those who may commit resources for conservation planning and implementation. For a conservation plan to be successful, the involvement of 
(1) Identify stakeholders for the planning region:

- Stakeholders include: (a) those who have decision-making powers; (b) those who will be affected by conservation plans for the region; (c) those with expertise about the region and (d) those who may commit resources for conservation plans;

- Include both local and global stakeholders;

- Ensure transparency in the involvement of all stakeholders from the beginning.

(2) Compile, assess, and refine biodiversity and socio-economic data for the region:

- Compile available geographical distribution data on as many biotic and environmental parameters as possible at every level of organization;

- Compile available socio-economic data, including values for alternate uses, resource ownership and infrastructure;

- Collect relevant new data to the extent feasible within available time; remote-sensing data should be easily accessible; systematic surveys at the level of species (or lower levels) will rarely be possible;

- Assess conservation status for biotic entities, for instance, their rarity, endemism and endangerment;

- Assess the reliability of the data, formally and informally; in particular, critically analyze the process of data selection;

- When data do not reflect representative samples of the landscape, correct for bias and model distributions.

(3) Identify biodiversity surrogates for the region:

- Choose true surrogate sets for biodiversity (representing general "biodiversity") for part of the region; be explicit about criteria used for this choice;

- Choose alternate estimator surrogate sets (for representing true surrogate sets in the planning process);

- Prioritize sites using true surrogate sets; prioritize sites using as many combinations of estimator surrogate sets as feasible and compare them;

- Potentially also use other methods of surrogacy analysis to assess estimator surrogate sets, including measures of spatial congruence between plans formulated using the true and estimator surrogate sets;

- Assess which estimator surrogate set is best on the basis of (a) economy and (b) representation.

(4) Establish conservation targets and goals:

- Set quantitative targets for surrogate coverage;

- Set quantitative targets for total network area;

- Set quantitative targets for minimum size for population, unit area, etc.;

- Set design criteria such as shape, size, dispersion, connectivity, alignment and replication;

- Set precise goals for criteria other than biodiversity, including socio-political criteria. 
Table 1.1 (cont.)

(5) Review the existing conservation-area network (CAN):

- Estimate the extent to which conservation targets and goals are met by the existing set of conservation areas;

- Determine the prognosis for the existing CAN;

- Refine the first estimate.

(6) Prioritize new areas for potential conservation action:

- Using principles such as complementarity, rarity and endemism, prioritize areas for their biodiversity content to create a set of potential conservation-area networks;

- Starting with the existing CAN, repeat the process of prioritization to compare results;

- Incorporate socio-political criteria, such as various costs, if desired, using a trade-off analysis;

- Incorporate design criteria such as shape, size, dispersion, connectivity, alignment and replication, if desired, using a trade-off analysis.

- Alternatively, carry out the last three steps using optimal algorithms.

(7) Assess prognosis for biodiversity within each newly selected area:

- Assess the likelihood of persistence of all biodiversity surrogates in all selected areas. This may include population viability analysis for as many species using as many models as feasible;

- Perform the best feasible habitat-based viability analysis to obtain a general assessment of the prognosis for all species in a potential conservation area;

- Assess vulnerability of a potential conservation area from external threats, using techniques such as risk analysis.

(8) Refine networks of areas selected for conservation action:

- Delete the presence of surrogates from potential conservation areas if the viability of that surrogate is not sufficiently high;

- Run the prioritization protocol again to prioritize potential conservation areas by biodiversity value;

- Incorporate design criteria such as shape, size, dispersion, connectivity, alignment and replication.

(9) Examine feasibility using multi-criteria analysis:

- Order each set of potential conservation areas by each of the criteria other than those used in Stage 6;

- Find all best solutions; discard all other solutions;

- Select one of the best solutions.

(10) Implement a conservation plan:

- Decide on most appropriate legal mode of protection for each targeted place;

- Decide on most appropriate mode of management for persistence of each targeted surrogate;

- If implementation is impossible return to Stage 5;

- Decide on a timeframe for implementation, depending on available resources.

(11) Periodically reassess the network:

- Set management goals in an appropriate timeframe for each protected area;

- Decide on indicators that will show whether goals are met;

- Periodically measure these indicators;

- Return to Stage 1. 
stakeholders should be transparent. Identifying and involving stakeholders can be a difficult and laborious process, but, if it is done properly, it can help mitigate threats to potential priority areas and improve the chances that conservation plans will be implemented (Wilson et al. 2005; see also Chapter 5, Example 5.5). Some recent planning exercises have emphasized that protocols and tools developed to aid planning need to be easy to use and transparent to stakeholders (Pierce et al. 2005; Knight et al. 2006; Rouget et al. 2006).

The second stage is data collection and treatment. This involves collating existing data, collecting new data if required, and any treatment of data that might be needed for subsequent use in conservation planning. The care and attention given this stage has a major bearing on the quality of the outcome. It can be time-consuming, labor intensive, and scientifically and technically challenging. However, the collection and treatment of the biological and environmental data are crucial components of systematic conservation planning. They can place severe constraints on the planning process if not done properly. We regard the collection and treatment of the biological and environmental data as so important that we have devoted two whole chapters to it (Chapters 3 and 4). Cost and the urgency to act tend to foster the use of existing data held, for example, in museums and herbariums or data that can be derived remotely, for example environmental data such as climate surfaces and other maps. All possible use should be made of such data. However, much greater attention than has been paid in the past should be devoted to the design of surveys to collect new biological records from the field (Margules \& Austin 1994; Haila \& Margules 1996). Field collections of species records are always being made by museums, herbariums, management agencies and others. Environmental stratification combined with recording the absence as well as the presence of species will deliver data sets that are comprehensive and consistent in detail across entire planning regions (Chapter 3). At this stage it is also desirable to compile as much social and economic data as possible, which may then be used in a trade-off analysis in Stage 6, or alternatively in a multi-criteria analysis at Stage 9 in Table 1.1. Socio-economic data include the expected monetary value of the natural resources in candidate conservation areas, or alternative measures such as timber volume or agricultural potential (as in Chapter 8, Section 8.5). They can also include human population density, cultural practices and preferences, including information on land ownership and tenure, as well as infrastructure.

The third stage is to choose biodiversity surrogates. This means selecting those features that are going to be used to represent biodiversity in the 
planning process. Taxa subsets, species assemblages, and environmental variables and classes, or combinations of two or more of these three, have all been used in conservation planning. Species or other features at risk, and rare or endemic species or features are obvious candidate surrogates. Note that social, cultural and economic significance have at times been just as important as biological significance in the choice of surrogates. Charismatic or iconic species and species with commercial value have often been used as biodiversity surrogates. The choice of surrogates is also a crucial stage and is discussed at length in Chapter 2. The choice of surrogates will always be constrained to a certain extent by what data are available, or realistically obtainable in an acceptable timeframe. Therefore, this choice is never independent of the results of the previous stage.

The fourth stage is to establish planning region goals and targets. The overall goals, identifying priority areas and separating them from threats, were identified above. At this stage, explicit targets for the representation of surrogates within a conservation-area network for the region of interest must be set. Without these targets it is impossible to determine the success or failure of a plan. Typical targets might be populations of a certain size, or a specified number of populations of species, or the spatial extent (percentage of coverage) of assemblages or environmental classes. Again, the actual numbers used here are often not determined, sometimes not even strongly suggested, by biological criteria such as models or empirical data. Viable population sizes are known for only a handful of species in a few habitats. Actual targets most often represent conventions arrived at by biological intuition, or a limited budget. Soulé and Sanjayan (1998) have argued that the achievement of such targets could provide an unwarranted sense of security, suggesting that biodiversity was being protected when in fact the targets have little or no biological meaning. This is undoubtedly true, but, as noted above, conservation areas are priority areas for the allocation of scarce management resources, and their identification is not supposed to provide a complete solution to the problem of protecting biodiversity. Priority areas alone will not protect biodiversity. Identifying priority areas is only one stage in the challenging, but necessary, task of learning to manage whole regions so that ecosystem processes and the biodiversity they give rise to can be sustained along with the generation of livelihoods.

At this stage it is also appropriate to introduce design criteria. Though not usually included in setting targets, these are ecological characteristics of the actual conservation areas - size, shape, dispersion, connectivity, alignment and replication, for example. In meeting conservation goals 
there seems no doubt that big is better than small, but ecology does not say how big is big enough. In addition, the roles of shape, dispersion, connectivity, alignment and replication in conservation-area networks remain controversial (Diamond 1975; Margules et al. 1982; Margules \& Pressey 2000). Biogeographical theory, successional pathways, space requirements (especially for wide-ranging species), source-sink population structures, and habitat modification all impact conservation area design (Chapter 6).

The fifth stage is a review of any existing conservation areas within the planning region. The purpose is to determine the extent to which conservation targets have already been met and therefore to identify gaps that need to be filled by new conservation areas. Usually, existing conservation areas have been established on land that has (or had at the time of establishment) little economic value. Gaps in existing conservation-area networks therefore are often in productive areas or close to population centers where competition for natural resources is highest. This emphasizes the need for flexibility in planning and signals the importance of cost trade-offs (Chapters 7 and 8). A careful assessment of the performance of existing conservation areas is critical because, in practice, conservation plans will typically consist of augmenting an existing network rather than creating one from scratch.

The sixth stage involves prioritizing new areas for conservation action to satisfy the targets and goals set in the fourth stage. This stage corresponds to what has previously been thought of as reserve network selection. It is discussed in detail in Chapter 5 and examples of how it has been done are worked through in Chapter 8 . The change of terminology recognizes the fact that designating reserves that exclude human habitation is only one of many possible conservation measures that could be implemented in biodiversity priority areas (Sarkar 2003). This stage is at the heart of systematic conservation planning. It implements complementarity as a measure of conservation value: one site has greater complementarity than another if it has more biodiversity features (species, assemblages, habitat types, etc.) that have not already met their representation target in the conservation area network. Some measures of complementarity also implement cost trade-offs (Faith \& Walker 2002; Faith et al. 2001a). This takes advantage of the fact that there are usually many spatial arrangements of selected areas in planning regions that each achieves the conservation goals. A set of conservation priority areas can be sought that optimizes opportunity costs such as agriculture, logging, recreation, industrial development and urbanization, but nevertheless achieves the conservation goal. Stage 9 below represents an alternative approach to 
taking costs and competing uses of biodiversity into account. In many practical applications, area selection will be limited to those areas that are not obviously irrelevant to conservation because of extensive habitat degradation, for instance, completely built-up areas. Thus, Stage 6 may include a preliminary exclusion of such areas.

The seventh stage assesses the risks to the persistence of biodiversity in selected areas. Threats can come from outside or within. Size, shape, dispersal, connectivity, alignment and replication are some ecological criteria for risk assessment. Suitability for competing uses such as agriculture or urban development increases the probability that a site will be lost to these land uses (Pressey \& Taffs 2001). Risk assessment is a difficult task and more remains uncertain than what is known. Chapter 6 considers how vulnerability and threat might be taken into account in conservation planning. Once risks to persistence have been assessed, it is likely that some areas with a poor prognosis will be dropped and prioritization repeated without those areas as candidates. It may be that there are no substitutes for an area with a poor prognosis and in that case a decision has to be made whether to spend potentially scarce management resources on intensive management actions to improve the prognosis or relinquish the biodiversity features of that site to their fate and divert management resources to areas and features with a greater likelihood of long-term persistence. The eighth stage then, is the reiteration of the prioritization process in Stage 6.

The ninth stage attempts to take account of competing uses of land other than biodiversity conservation such as agriculture, recreation, etc. Typically, a number of sets of selected areas (or "solutions" from an area prioritization algorithm) are first produced with each satisfying the biodiversity representation targets. Stakeholders decide the relative importance of different potential uses of land, and these preferences are used to order each of the alternative solutions by all the criteria other than biodiversity. Biological criteria other than representation of surrogates, such as size, shape, dispersal, connectivity, alignment and replication, can also be incorporated in this way through multi-criteria analysis. The best solutions become candidates for implementation and the others are discarded. There are a variety of techniques for carrying out such a multi-criteria analysis, mainly developed by economists and the decisiontheory community and only lately being explicitly incorporated into conservation planning (Moffett \& Sarkar 2005). The most useful techniques of multi-criteria analysis are discussed in Chapter 7. If the alternative of incorporating trade-offs in the area selection process (Stage 6) 
is preferred, and all relevant criteria have been incorporated into the trade-off analysis, then this stage is redundant.

The tenth stage is implementation of the conservation plan. This requires decisions on the most appropriate form of legal protection for each selected area and the most appropriate management actions for each selected area. An important consideration here is the scheduling of implementation. Resources are not normally available to act on all selected areas simultaneously. More vulnerable areas might receive priority, especially if the features they contain are absent or scarce in other areas (Pressey \& Taffs 2001; Wilson et al. 2005). If it proves impossible to implement the plan because, for example, some areas are seriously degraded, budgets have changed or the forgone opportunity costs associated with parts of the plan are unacceptable to society, then it is necessary to return to Stage 6 and try again. Because of this inevitable scheduling problem we must accept that planning is a dynamic iterative process. Planners and policy-makers should return to earlier stages repeatedly because social and economic conditions change, social and political attitudes change, and knowledge accumulates. The plan that was right given the knowledge base and the social, economic and political climate last year will not necessarily be the best plan this year.

The eleventh and final stage is to monitor the effectiveness of management actions in sustaining the features that areas were selected for. Monitoring also requires that thresholds are defined, which, if passed, warn that unacceptable changes might be underway. The status of biological entities changes over time, as do social and economic conditions. Management actions that seemed appropriate at one point in time might be less effective at another point in time. Changes to management prescriptions are one response. As suggested above, another response might be to repeat the entire conservation-planning process periodically. The most desirable situation is that conservation planners have the facility to repeat the process as and when needed in order to take account of societal change and the gaining of new knowledge. Such iterative, dynamic managerial response with feedback is called "adaptive management" (Holling 1978).

\subsection{Summary}

The goal of systematic conservation planning is to identify areas that should have priority for the allocation of scarce biodiversity-management resources and to separate those areas from factors that threaten their 
persistence. Biodiversity is a complex concept that is impossible to estimate or quantify completely. For planning purposes we have to use partial measures or biodiversity surrogates that can be quantified and assessed in the field, for example taxa subsets, habitat types, etc. A key concept in systematic conservation planning is complementarity; the marginal contribution an area makes to representing the full set of attributes chosen as biodiversity surrogates.

Systematic conservation planning is a structured approach with feedback and reiteration, where needed, at any stage. In this book we recognize 11 distinct stages. They range from the engagement of people who influence, use and manage biodiversity in the planning region, through choices about how biodiversity is to be measured and mapped, to setting biodiversity goals that can be translated into quantifiable targets, estimating the extent to which targets have been met in existing conservation areas, using complementarity to identify new priority conservation areas, implementing planning choices on the ground and monitoring management actions to ensure the continued contribution by chosen areas to the conservation goal. 